\title{
AVALIAÇÃO DA QUALIDADE DE VIDA DOS USUÁRIOS DE UM NÚCLEO DE APOIO À SAÚDE DA FAMÍLIA - NASF
}

DOI: $10.22289 / 2446-922 X . V 3 N 1 A 5$

\author{
Rildo Jose Totoro \\ Michelle Dayanne Sousa \\ Raphael Cezar Carvalho Martins \\ Eduardo Paul Chacur \\ Fernando Leonardo Diniz Souza ${ }^{1}$
}

\section{RESUMO}

A criação dos Núcleos de Apoio à Saúde da Família (NASF) marca de maneira definitiva a atuação de equipes multidisciplinares no âmbito do Sistema Único de Saúde (SUS). O presente estudo tem como objetivo comparar a qualidade de vida de usuários e indivíduos não usuários do NASF por meio do questionário genérico SF36. Trata-se de um estudo quantitativo transversal, no qual os questionários foram aplicados em dois grupos de trinta usuários cada, participantes e não participantes das atividades do NASF em um município de porte médio de Minas Gerais. Os resultados demonstraram que somente no domínio do questionário, Limitação por Aspectos Físicos (LF), foram encontradas diferenças significativas para $p<0,001$. Conclui-se que o NASF deve direcionar com mais amplitude suas atividades multidisciplinares para que todos os aspectos que interferem na qualidade de vida dos indivíduos, não só os aspectos físicos, tenham impacto positivo.

Palavras-chaves: NASF. Qualidade de vida. Questionário SF 36.

\section{ABSTRACT}

The creation of the Family Health Support Centers (NASF) definitively marks the performance of multidisciplinary teams within the scope of the Unified Health System (SUS). The present study aims to compare the quality of life of non-users and users of NASF through the SF36 generic questionnaire. This is a cross-sectional quantitative study, in which the questionnaires were applied to two groups of thirty users, divided between participants and non-participants of NASF activities, in a medium-sized municipality of Minas Gerais. The results showed that only in the domain Limitation by Physical Aspects (LF) significant differences were found for $p<0,001$. It's concluded that the NASF should direct its multidisciplinary activities so that all the aspects that interfere in the quality of life the individuals have positive impact.

Keywords: NASF. Quality of life. Questionnaire SF 36.

\footnotetext{
${ }^{1}$ Endereço eletrônico de contato: drfernandodiniz@gmail.com
}

Recebido em 12/06/2017. Aprovado pelo Conselho Editorial e aceito para publicação em 13/06/2017.

Rev. Psicol Saúde e Debate. Jan., 2017:3(1):50-67. 


\section{INTRODUÇÃO}

O crescimento no número de pessoas que apresentam morbidades pela evolução da transição epidemiológica vem aumentando em todo o mundo, esse processo se deve ao aumento da expectativa de vida da população devido ao avanço das práticas em saúde, com isso o envelhecimento e seus aspectos especiais de saúde requerem maior preocupação quanto ao envelhecer mais saudável, e com qualidade de vida. ${ }^{(1)}$

Apesar de não haver uma única definição específica para Qualidade de Vida (QV) algumas delas merecem destaque, como a da Organização Mundial de Saúde (1998) que define qualidade de vida como. O entendimento que o indivíduo tem de sua posição na vida dentro do contexto de sua cultura e do sistema de valores de onde vive, e em relação a seus objetivos, expectativas, metas, padrões e preocupações. ${ }^{(2,3)}$ É um conceito muito amplo que incorpora de uma maneira complexa a saúde física de um individuo, seu estado psicológico, seu nível de dependência, suas relações sociais, suas crenças e sua relação com características proeminentes no ambiente. ${ }^{(4)}$

Com a finalidade de melhorar a situação da população brasileira diante a desigualdade na assistência à saúde, o movimento de Reforma Sanitária buscou democratizar o oferecimento de serviços de saúde através da oferta integral e contínua. As propostas do movimento culminaram na criação do Sistema Único de Saúde (SUS) que incorporou na Constituição Federal de 1988 a maior parte das ideias apresentadas na VIII Conferência Nacional de Saúde em 1986. O SUS foi regulamentado pelas Leis $n^{\circ}$ 8080/90 e $n^{\circ}$ 8142/90 pautado pelos pilares da universalidade e integralidade do acesso além da participação popular por meio de conselhos estabelecidos nas três esferas de poder. ${ }^{(5,25,26)}$ Além disso, as ações propostas derivam da atenção primária à saúde na tentativa de antecipar agravos e suas complicações.

Em 1994, após a consolidação do SUS, foi criada a hoje chamada, Estratégia Saúde da Família (ESF), a qual apresenta objetivos de reorganizar a prática assistencial, entendendo a família como objeto principal e a interação da mesma com o ambiente onde vive. ${ }^{(5)}$ 
Com a necessidade de atender e gerenciar todas as demandas encontradas no espaço vivo da ESF, objetivando a melhoria da qualidade, eficácia e sua eficiência da Atenção Básica em Saúde (ABS), foram criados em 24 Janeiro de 2008, através da Portaria GM/MS no154 os Núcleos de Apoio à Saúde da Família (NASF), os quais são definidos por equipes formadas por diferentes profissionais, de varias áreas que atuam em parceria com a ESF. A partir dessa portaria criaram-se dois tipos de NASF: modalidade - 1: que deve ser composto por no mínimo cinco categorias profissionais de nível superior: Médicos com pelo menos uma destas especialidades, Acupunturista, Ginecologista, Homeopata, Pediatra, ou Psiquiatra, e profissionais de outras áreas de atuação como: Assistente Social, Educador físico, Farmacêutico, Fisioterapeuta, Fonoaudiólogo, Nutricionista, Psicólogo, e Terapeuta Ocupacional. ${ }^{(6)}$ Este NASF deve realizar suas atividades em no mínimo oito ESF e no máximo em vinte ESF. Modalidade - 2: que pode ser formado por no mínimo 3 três categorias de profissionais: Assistente Social, Educador Físico, Farmacêutico, Fisioterapeuta, Fonoaudiólogo, Nutricionista, Psicólogo e Terapeuta Ocupacional, sendo que para esta modalidade e permitida apenas para aqueles municípios que tenham menos de 100 mil habitantes ou que tenham uma densidade de sua população abaixo de $10 \mathrm{dez}$ habitantes por quilômetro quadrado. $\left.{ }^{6}\right)$ Segundo o Ministério da Saúde (2008), os NASF devem funcionar em horário de trabalho igual com o das ESF, e que a carga horária dos profissionais que integram o NASF.

Em 2010, a Portaria $N^{\circ} 2.843$, de 20 setembro, cria no âmbito do SUS, mais uma modalidade a $3 \mathrm{com}$ a principal prioridade em promover a atenção integral em saúde e saúde mental aos usuários de crack, álcool e outras drogas na Atenção Básica para municípios com porte populacional menor que 20.000 habitantes, realizando suas atividades em no mínimo 4 e no máximo 7 ESF e ter no mínimo 3 profissionais de nível superior de ocupações não coincidentes com as categorias profissionais descritas na Portaria GM/MS n.154 de 2008 do Ministério da Saúde em $2010 . \cdot^{(7,10)}$

Com objetivo de avaliar a eficácia das atividades proposta pelos NASF, surge a necessidade de efetuar estudos perante os usuários dos NASF para verificar a efetividade das intervenções e o seu impacto na qualidade de vida destes indivíduos. 
Estudos sobre avaliação da qualidade de vida podem identificar o impacto causado por intervenções multiprofissionais em grupos de indivíduos expostos a tais ações. A ferramenta SF-36 é uma adaptação do questionário Medical Outcomes Study 36 - Item short form health survey, no Brasil ele foi estudado, traduzido e validado por Ciconelli(8). Martinez ${ }^{(9)}$ expõem que a versão em português do SF-36 é um questionário genérico, com conceitos não específicos, elaborado para uma determinada idade, doença ou grupo de tratamento e que permite diferentes comparações entre diversas patologias e entre diferentes formas de tratamentos. ${ }^{(9)}$

A ferramenta em questão e um questionário multidimensional formado e elaborado por 36 itens, divididos em 8 domínios: capacidade funcional (CF) formado por dez 10 itens, limitações por aspectos físicos (LF) formado por quatro 4 itens, dor (DR) formado por dois 2 itens, estado geral de saúde (ES) formado por cinco 5 itens, vitalidade (VTA) formado por quatro 4 itens, aspectos sociais (AS) formado por dois 2 itens, limitação por aspectos emocionais (LE) formado por três 3 itens, saúde mental (SM) formado por cinco 5 itens, e mais uma questão que avalia e compara as condições de saúde atual com a de um ano atrás ${ }^{(8)}$. O questionário tem como objetivo avaliar tanto aspectos negativos de saúde do individuo entrevistado tipos de doença ou enfermidade, como seus aspectos positivos deste mesmo individuo (bem-estar). ${ }^{(8)}$ Todos os dados são avaliados a partir da transformação de todas as respostas em escores que variam em uma escala de 0 a 100 para cada componente, não havendo um único valor especifico que resuma toda a avaliação do questionário, resultando em uma condição geral de saúde melhor ou pior. ${ }^{(8,24)}$ Este tipo de ferramenta se torna útil para verificar a efetividade de propostas que visam modificar a qualidade de vida de indivíduos, tais como, as propostas apresentadas pelos NASF Núcleos de Apoio à Saúde da Família.

Apesar de já terem sido realizados alguns estudos sobre a atuação dos Núcleos de Apoio à Saúde da Família, estudos referentes à qualidade de vida de pessoas que participam e não participam das atividades oferecidas pelo NASF ainda são escassos na literatura. Este estudo justifica-se pela necessidade de quantificar os impactos na qualidade de vida dos usuários dos NASF e compará-los com níveis de qualidade de vida de indivíduos inerentes ao programa. Sendo assim, o objetivo deste estudo é avaliar a qualidade de vida de indivíduos participantes e não 
participantes das atividades propostas pelos Núcleos de Apoio à Saúde da Família.

\section{MÉTODO}

Trata-se de um estudo quantitativo descritivo de caráter transversal com utilização da ferramenta SF 36 (Medical Outcomes Study 36 -Item Short-Form Health Survey), validado e traduzido para o português ${ }^{(8)}$. O SF 36 é um questionário com conceitos genéricos e não específicos, que permite comparações entre diferentes situações patológicas e entre diversos tipos de tratamentos ou intervenções, ele quantifica a qualidade de vida de indivíduos ou coletividades em determinado momento, a visão dos indivíduos quanto ao seu próprio estado de saúde contemplando os aspectos mais representativos sobre saúde em geral.(9) 0 questionário é formado por 36 itens divididos em oito domínios, na qual analisa aspectos distintos: ${ }^{(11,13)}$

1. Capacidade funcional (CF) elaborado por dez (10) itens: avalia se a presença e extensão das limitações impostas à capacidade física.

2. Limitações por aspectos físicos (LF), elaborado por dois (2) por itens.

3. Dor (DR) composto por dois (2) itens.

4. Estado geral de saúde (ES) composto por cinco (5) itens.

5. Vitalidade (VTA), formado por quatro (4) itens.

6. Aspectos sociais, composto por dois (2) itens: este domínio tem como objetivo analisar a integração e convívio do indivíduo em atividades sociais.

7. Limitações por aspectos emocionais, (LE) formado por três (3) itens.

8. Saúde mental (SM) composto por cinco (5) itens: este domínio tem como objetivo investigar as dimensões de depressão, ansiedade e se há alterações do comportamento.

Cada escala recebe um escore que varia de zero a cem onde 0 corresponde ao pior estado de saúde e 100 o melhor estado de saúde. ${ }^{(8)}$

O cálculo do SF-36 foi elaborado convertendo a maioria das questões em 8 oito domínios, sendo que para cada um dos domínios existe um cálculo diferente que varia de zero a cem. Resultado este chamado de Raw Scale porque o seu valor final não apresenta nenhum tipo de unidade em medida. 
A $2^{\circ}$ questão não faz parte do cálculo de nenhum domínio, sendo utilizado somente para avaliar o estado geral e o quanto o indivíduo está melhor ou pior comparado á 12 meses atrás. ${ }^{(8)}$

\section{AMOSTRA:}

Participantes: Neste estudo participaram 60 indivíduos, destes 30 indivíduos eram participantes de pelo menos uma das atividades oferecidas pelo NASF, e 30 indivíduos não faziam parte de nenhuma das atividades oferecidas pelo NASF. A maioria das amostras e formada por participantes do sexo feminino (80\%), com uma media de idade de 55 anos. E a minoria do sexo masculino (20\%), com media de idade de 57 anos.

Material: Os dados pessoais foram colhidos por meio de uma ficha de identificação que incluiu questões com nome, RG, CPF, data de nascimento, idade, sexo, endereço, telefone, função exercida no trabalho, quanto tempo exerceu esta função, qual função atual, quanto tempo exerce esta função.

O questionário (Medical Outcomes Study 36 -Item Short-Form Health Survey),(SF 36) validado e traduzido para o português, foi utilizado para avaliar a qualidade de vida (QV) dos 2 grupos pesquisados as dimensões medidas são: a capacidade funcional (CF), limitações por aspectos físicos (LF), dor (DR), estado geral de saúde (ES), vitalidade (VTA), aspectos sociais (AS), limitações por aspectos emocionais (LE), saúde mental (SM). Esses domínios podem ser agregados em duas medidas sumarias a física (MSF), e a mental (MSM) o valor varia de 0 a 100 , onde 0 e o pior estado de saúde e 100 o melhor estado de saúde.

Procedimento: O estudo foi realizado entre julho de 2016 e setembro 2016 com os grupos do NASF e os grupos de não NASF do Município, a pesquisa foi realizada em 2 fases $1^{\circ}$ fase para participantes do NASF, e a $2^{\circ}$ fase para não participantes do NASF. Os critérios para inclusão foram as seguintes: possuir aptidões para responder o questionário, indivíduos de ambos os sexos, raça ou religião. O critério para exclusão foi á presença de demência dos participantes, esta pesquisa faz parte de um estudo quantitativo descritivo de caráter transversal. Para testar as diferenças de acordo com o nível de qualidade de vida (QV), dos 2 grupos 
analisados foi utilizado o teste de (Kolmogorov-Smirnov) do programa SPSS Statistics versão 22.

Cenário do estudo: $O$ estudo foi realizado em um município de porte médio do estado de Minas Gerais, com atividade econômica predominante agrária, apresenta cerca de 148.000 habitantes e IDH de 0,765 . Após aprovação do projeto pelo comitê de ética e pesquisa, foram selecionados dois grupos de voluntários com 30 indivíduos cada um. O grupo denominado 1 , será composto de usuários de pelo menos uma atividade proposta pelo NASF do município. O grupo denominado 2 , será composto por indivíduos de mesma faixa etária do grupo 1, porém, não participantes de nenhuma atividade proposta pelo NASF.

Os questionários SF 36 foram aplicados inicialmente ao grupo 1, onde foi descrito o perfil etário desses indivíduos. Após a aplicação no grupo 1 foram selecionados indivíduos de perfil etário semelhante, grupo 2, nos quais foram aplicados o mesmo questionário.

O responsável pela pesquisa obteve junto á secretaria de saúde do município a documentação que o autorizou para aplicação dos questionários referidos bem como o oferecimento de Termos de Consentimento Livre e Esclarecido, de acordo com a Resolução 466/12 do Conselho Nacional de Saúde (CNE), podendo os voluntários se desligar a qualquer momento da pesquisa e assegurando o sigilo dos dados pessoais. Concepções estas aprovadas pelo Comitê de Ética e Pesquisa da Faculdade Patos de Minas sob o parecer n 1.759.633.

$O$ estudo foi direcionado inicialmente somente a moradores que vivem no município em seu perímetro urbano. Os dados dos dois grupos foram comparados estatisticamente, dentro dos padrões de escore do questionário utilizado. 


\section{RESULTADOS}

Tabela 1: teste de variância entre os grupos analisados.

\begin{tabular}{|ll|r|r|r|}
\hline \multirow{2}{*}{ Grupo pesquisado } & & \multicolumn{3}{|c|}{ Kolmogorov-Smirnov } \\
\cline { 3 - 5 } & NASF &, 205 & $n$ & $\mathrm{p}$ \\
\hline Capacidade funcional & NÃO NASF &, 110 & 30 &, 002 \\
& Limitação por aspectos &, 216 & 30 &, 200 \\
\hline físicos & NÃO NASF &, 223 & 30 &, 001 \\
\hline Dor & NASF &, 101 & 30 &, 001 \\
& NÃO NASF &, 187 & 30 &, 200 \\
\hline Estado geral de saúde & NASF &, 177 & 30 &, 009 \\
& NÃO NASF &, 181 & 30 &, 017 \\
\hline Vitalidade & NASF &, 155 & 30 &, 013 \\
& NÃO NASF &, 171 & 30 &, 065 \\
\hline Aspectos sociais & NASF &, 370 & 30 &, 025 \\
& NÃO NASF &, 375 & 30 &, 000 \\
\hline Limitação por aspectos & NASF &, 376 & 30 &, 000 \\
emocionais & NÃO NASF &, 263 & 30 &, 000 \\
\hline Saúde mental & NASF &, 146 & 30 &, 000 \\
& NÃO NASF &, 156 & 30 &, 104 \\
& & & 30 &, 059 \\
\hline
\end{tabular}

Caracterização da Qualidade de vida (QV), de participantes versus não participantes das atividades oferecidas pelo NASF. Não apresentaram níveis de significância em relação á medida sumária mental (MSM) e a medida sumaria física (MSF) entre os 2 grupos avaliados.

A análise multivariada dos dados revelou que existem diferenças significativas na (QV) de apenas um domínio o de limitação por aspectos físicos (LF), esta analise foi favorável ao grupo de indivíduos que participam de pelo menos uma das atividades oferecida pelo NASF, onde o seu resultado foi $p<0,001$ e seu domínio 216 para participantes do NASF, versus $p<0,001$ e seu domínio 223 para não participantes do NASF.

Contudo, não se verificou diferenças na (QV), em 7 domínios que foram os de capacidade funcional (CF), onde $p<0,002$ e seu domínio 205 para o grupo 1 que são os de participantes do NASF, versus $p<0,200$ e seu domínio 110 para o grupo 2 que não são participantes do NASF.

Rev. Psicol Saúde e Debate. Jan., 2017:3(1):50-67. 
No domínio dor (DR), também não houve diferenças onde $p<0,200$ e seu domínio 101 para o grupo 1 versus p<0,009 e domínio 187 para o grupo 2.

No $4^{\circ}$ domínio estado geral de saúde (ES), a tabela demonstra o seguinte resultado para o grupo 1 p<017 e seu domínio 177 já no grupo 2 o resultado ficou em $\mathrm{p}<0,13$ e seu domínio 181.

O domínio vitalidade (VTA), ficou da seguinte forma $p<0,065$ e seu domínio 155 para o grupo 1 versus $p<025$ e seu domínio 171 para o grupo 2.

Em aspectos sociais (AS), também não houve significância onde $p<000$ e seu domínio 370 para o grupo 1 e p<000 e seu domínio 375 para o grupo 2.

O domínio de limitação por aspectos emocionais (LE), ficou em $p<000$ e seu domínio 376 para o grupo 1 versus p<000 e seu domínio 273 para o grupo 2.

No último domínio o de saúde mental (SM), ficou da seguinte forma $p<104$ e 0 seu domínio 146 para o grupo 1 versus p<0,059 e seu domínio 156.

\section{DISCUSSÃO}

A tabela acima (tabela1) descreve os resultados de variância dos grupos analisados o teste escolhido para á analise desta pesquisa foi o teste de Kolmogorov-Smirnov.

Em estatística, o teste Kolmogorov-Smirnov e amplamente sugerido para analise de pequenos grupos, é usado para determinar se duas distribuições de probabilidade subjacentes diferem uma da outra ou se uma das distribuições de probabilidade subjacentes difere na distribuição em hipótese, em qualquer dos casos com base em amostras finitas Este teste não requer a estimação da variância e da média da população são testes que podem ser usados tanto para variáveis nominais como para variáveis ordinais ou quantitativa. O teste Kolmogorov-Smirnov se baseado na função de distribuição empírica (FDE) dos dados, apresenta vantagens sobre outros testes de aderência, incluindo maior poder e invariância em relação aos pontos médios dos intervalos escolhidos. ${ }^{(14)}$

A seguir descrições mais detalhadas desta pesquisa e de cada um dos domínios do SF-36 será apresentada, onde significância estatística demostrar um 
resultado de <0,001 seguida de comparações com outros estudos envolvendo pesquisas em que o questionário SF-36 foi utilizado.

\section{Capacidade Funcional (CF)}

O domínio CF, é formado por dez questões, relacionados a dificuldades de realização de atividades diárias devido à saúde, a capacidade funcional (CF) tem como principal objetivo avaliar tanto a presença quanto, a extensão das limitações com relação à condição física de cada individua entrevistado.

Nesta pesquisa, os valores de escores encontrados, para este domínio foi de p<0,002 e seu domínio 205 para o grupo 1 que são os de participantes do NASF, versus $p<0,200$ e seu domínio 110 para o grupo 2 que não são participantes do NASF. Valores estes sem significância estatística para este domínio.

No estudo de Silqueira(15) os resultados foram similares á esta pesquisa, neste domínio os valores de escores obtidos também foram baixos, por outro lado Alves $^{(16)}$ obteve maiores valores nesse domínio, para Gontijo(17) os resultados obtidos também foram favoráveis a este domínio, Ciconelli ${ }^{(8)}$ em sua pesquisa afirma que teve um melhor resultado para este domínio comparado aos outros domínios do questionário Health Assessment Questionaire (HAQ) questionário este usado como comparativo para avaliar este domínio o qual não obteve valores de significância ao contrario do SF 36 que teve valores de significância para este domínio valor este que foi: $p<0,001$.

Jesus $^{(18)}$ realizou uma pesquisa semelhante a esta onde os resultados revelarão significância estatisticamente favorável para este domínio, onde os indivíduos que participavam de atividades físicas regulares obtiveram resultados de escores maiores que os indivíduos que não participavam de exercícios físicos regularmente, fato se que assemelha a outro estudo, Pimenta(19) também relatam em seus estudos, que a prática de exercícios físicos regulares apresentarão diferença, com significância estatística favorável para este domínio apenas em indivíduos que participavam de atividades físicas regularmente. 


\section{Limitação Aspectos Físicos (LF)}

Este domínio e formado por quatro itens relacionados a problemas com alguma atividade ou trabalho feito diariamente em decorrência de sua saúde física, o domínio aspectos físicos tem como objetivo avaliar o quanto essas limitações influenciam a vida diária do paciente dificultando as suas atividades regulares. ${ }^{(12)}$

Em relação aos escores encontrados, neste estudo os valores obtidos foram favoráveis para este domínio os resultados encontrados demonstraram significância estatística entre os dois grupos avaliados resultados estes que evidenciaram um nível superior de (LF) para o grupo 1 em relação ao grupo 2 os escores deste domínio ficaram em $\mathrm{p}<0,001$ e seu domínio 216 para participantes do NASF, versus $\mathrm{p}<0,001$ e seu domínio 223 para não participantes do NASF.

Resultados estes que se assemelham a outros estudos Farinha ${ }^{(20)}$ descreve em seu estudo que os valores de significância foram favoráveis a este domínio entre os grupos analisados, para Silva(21) relata em seu estudo resultados significantes para este domínio comparado aos outros domínios.

No estudo de Jesus ${ }^{(18)}$ os resultados de significância demonstraram valores de escores favoráveis a este domínio valores estes que ficaram desta forma, média de 76,66 e um desvio padrão de 25,81. Resultados estes que comprovam que a soma dos escores do grupo de atividades físicas e superior ao do grupo sem atividade física. Por outro lado, Ciconelli(8) descreve em seu estudo uma soma e variáveis diferentes para este domínio, a soma de suas variáveis foi de $p<0,8468$ confirmando que para este domínio a soma de escores ficou abaixo dos níveis de significância estatística.

Resultados estes semelhantes ao de Suzano(22) o valor obtido para este domínio em sua pesquisa revelou uma soma de escores baixos valor este que foi 48,4 ou $\mathrm{p}<0,048$ ficando abaixo dos níveis de significância estatística. Estes resultados também se assemelham ao estudo de Castro(23) os resultados do seu estudo e pesquisa evidenciaram comprometimento nas diferentes dimensões analisadas pelo questionário SF-36, sendo que os menores valores médios observados foi nas dimensões aspectos físicos(LF) e vitalidade(VT). 


\section{Dor (DR) e Estado Geral de Saúde (ES)}

Dor é composto por dois itens á escala dor tem como objetivo avaliar a sua proporção e se esta é causa a gera de alguma forma algum tipo de limitação à vida diária do paciente.

Estado geral de saúde (ES) e composto por cinco itens o estado geral de saúde tem como objetivo avaliar a saúde do paciente em um modo geral em suas, atividades diárias.

Neste estudo em relação a estes dois domínios os valores de soma de escores não obtiveram resultados de variância de significância estatística sendo que os valores desses dois domínios ficaram abaixo das somas de variáveis entre os dois grupos pesquisados valores estes que são: para dor (DR) $p<0,200$ e seu domínio 101 para o grupo 1 versus p<0,009 e domínio 187 para o grupo 2 .

Para estado geral de saúde (ES) os valores são: para o grupo 1 p<0,017 e seu domínio 177 já no grupo 2 o resultado ficou em $\mathrm{p}<0,013$ e seu domínio 181 . Lembrando que para haver significância estatística os valores de $p$ deveram ser de $<0,001$.

Este resultado contradiz outros estudos que avaliaram estes dois domínios através do questionário SF 36, para Jesus ${ }^{(18)}$ em seu estudo, foram encontradas diferenças estatisticamente significativas entre aqueles que praticam atividade física e aqueles que não praticam os que praticam atividades físicas obtiveram uma soma melhor nos dois domínios Dor (DR) e Estado Geral de Saúde (ES), em relação aos que não praticam atividades físicas, Suzano ${ }^{(22)}$ também relata em seu estudo uma soma relevante e de significância estatística ao domínio estado geral de saúde (ES) valor este de $(63,8)$, porem o domínio dor (DR) teve sua soma de escore abaixo do nível desejado valor este de $(36,9)$.

Para Silva(21) em seu estudo que avaliou dois grupos de pessoas um dos grupos participantes de dança, de salão e outro grupo de hidroginástica as somas para estes dois domínios obtiveram relevância estatísticas semelhantes para os dois grupos analisados. 
Por outro lado o estudo de Pimenta(19) teve resultados semelhantes ao deste estudo a soma dos escores não evidencio relevância significativa estatisticamente para estes dois domínios.

\section{Vitalidade (VTA)}

Este domínio é formado por quatro (4) itens relacionados a vigor, esgotamento, energia e cansaço estes itens avaliam qual o nível de energia e de fadiga, como a disposição para executar novas tarefas diárias.

Para este domínio neste estudo os resultados dos valores da soma de escores não evidencio resultados estatísticos entre os dois grupos avaliados resultados este que, ficou da seguinte forma $p<0,065$ e seu domínio 155 para 0 grupo 1 versus $p<0,025$ e seu domínio 171 para o grupo 2 .

Resultados semelhantes foram analisados em outras literaturas Silqueira(15) obteve neste domínio o menor escore entre todos os outros domínios analisados em seu estudo somas estas que são de 64,7 com desvio padrão de $<0,021$. Castro(23) obteve resultados semelhantes a esta pesquisa em seu estudo o nível de significância para este domínio (VTA) e a soma dos escores também ficaram abaixo dos outros domínios. Pimenta(19) não encontrou diferenças significativas neste domínio entre os grupos avaliados em sua pesquisa, já para Silva(21) apresentou um resultado positivo para este domínio com soma de escores favoráveis a (VTA) resultado semelhante ao de Farinha(20) neste domínio o seu estudo também apresentou somas com significância estatística para (VTA).

\section{Aspectos Sociais (AS)}

Aspectos Sociais (AS) este domínio e composto por dois itens relacionados a quanto o problema físico ou emocional interfere nas atividades sociais normais do dia a dia do paciente estes dois itens tem como objetivo avaliar a integração do paciente com atividades sociais.

Entre os dois grupos analisados neste estudo não houve resultados de significâncias estatísticas para este domínio os resultados de escores e de variância 
não produziram resultados favoráveis para este domínio, onde $p<000$ e seu domínio 370 para o grupo 1 e p<000 e seu domínio 375 para o grupo 2.

Resultado este que não se corrobora com o estudo de Silqueira(15) em sua pesquisa este domínio foi o que apresentou maior escore. Em relação aos escores encontrados, o valor para (AS) foi de $p<0,001$, Castro(23) obteve em seu estudo resultados favoráveis com significância estatísticas para este domínio com 0 resultado de $p<0,004$ Alves $^{(16)}$ também obteve valores maiores para este domínio Gontijo $^{(20)}$ através de sua pesquisa também chegou a soma de bons resultados para este domínio com uma variância de $\mathrm{p}=0,007$.

\section{Limitações por Aspectos Emocionais (IE) e Saúde mental (SM)}

O domínio (IE) é composto por três itens relacionados às alterações com o trabalho ou algumas atividades feitas diariamente em decorrência de problemas emocionais.

SM é composto por cinco itens este domínio tem como objetivo avaliar a saúde mental verificar a ansiedade, alterações do comportamento ou descontrole emocional e bem-estar psicológico como depressão e nervosismo.

Para este estudo depois da soma de escores e dados analisados o mesmo não obteve somas com significâncias estatísticas para estes dois domínios dados estes que foram: para limitação por aspectos emocionais (LE), $p<000$ e seu domínio 376 para o grupo 1 versus $p<000$ e seu domínio 273 para o grupo 2.

E para saúde mental (SM), ficou da seguinte forma $p<0,104$ e o seu domínio 146 para o grupo 1 versus $p<0,059$ e seu domínio 156.

Dados estes que não corroboram com estudos de Farinha. ${ }^{(20)}$ onde ele relata dados positivos e com significância para este dois domínios.

Entanto para Gontijo ${ }^{(17)}$ os seus resultados foram de $<0,007$ sem significância estatística para (IE) enquanto para (SM) com significância estatísticas <0,001. 


\section{CONSIDERAÇÕES FINAIS}

A comparação da qualidade de vida de indivíduos que participam de atividades que são oferecidas pelo NASF e dos que não participam, mostrou-se significativamente diferente somente no domínio limitação por aspectos físicos. Tal resultado sugere que atividades físicas regulares podem ter impacto positivo na qualidade de vida dos indivíduos. A ausência de relevância nos outros domínios sugere, porém, que as atividades do NASF não referentes diretamente à atividade física, devem se direcionar melhor pois o NASF é composto por equipes multidisciplinares que tem por objetivo atingir holisticamente $\mathrm{o}$ indivíduo e suas coletividades. 


\section{REFERÊNCIAS}

1 Paschoal SMP. Qualidade de vida do idoso: elaboração de um instrumento que privilegia sua opinião. [Dissertação de Mestrado]. São Paulo (SP): Universidade de São Paulo, Faculdade de medicina; 2000.

2 Bettarello PA, Saut TB. Análise dos resultados do questionário sobre a qualidade de vida - SF 36, após aplicação dom lian gong em 18 terapias e ginástica laboral, em funcionários do setor de editoração do centro universitário claretiano de batatais. [Trabalho de Conclusão de Curso]. Batatais (SP): Centro Universitário Claretiano de Batatais, Graduação em Fisioterapia; 2006.

3 Teixeira ACP, Fonseca AR, Maximo IMNS. Inventário SF36: avaliação da qualidade de vida dos alunos do Curso de Psicologia do Centro UNISAL - U.E. de Lorena (SP). Psic: Rev de Psicol da Vetor Editora. 2002:3(1):16-27.

4 Miranda LC, Banhato EFC. Qualidade de vida na terceira idade: a influência da participação em grupos. Psicol em Pesquisa. 2008(1):69-80.

5 Franco TB, Merhy EE. Programa de Saúde da Família (PSF): Contradições de um Programa destinado à mudança do modelo tecnoassistencial. In: Merhy EE, et al. $\mathrm{O}$ trabalho em saúde: olhando e experienciando o SUS no cotidiano. São Paulo: Hucitec; 2004.

6 Barbosa EG, Ferreira DLS, Furbino SAR. Experiência da fisioterapia no Núcleo de Apoio à Saúde da Família em Governador Valadares, MG. Fisioter Mov. 2010:23(2):323-330.

7 BRASIL. Ministério da Saúde. Secretaria de Atenção à Saúde. Departamento de Atenção Básica. Diretrizes do NASF: Núcleo de Apoio a Saúde da Família. Ministério da Saúde, Secretaria de Atenção à Saúde, Departamento de Atenção Básica. Brasília : Ministério da Saúde, 2010.

8 Ciconelli RM, Ferraz MB, Quaresma MR. Tradução para a língua portuguesa e validação do questionário genérico de avaliação de qualidade de vida sf-36. Rev. Bras. Reumatol. 1999:39(3):1-10. 
9 Martinez MC, Paraguay MC, Latorre MRD. Relação entre satisfação com aspectos psicossociais e saúde dos trabalhadores. Rev Saúde Pública. 2004:38(1):55-61.

10 Souza FLD, et al . Implantação do Núcleo de Apoio à Saúde da Família: percepção do usuário. Saúde debate. 2013:37(97):233-240.

11 Orlando MM, Silva MSP, Lombardi Junior I. The influence of the practice of physical activity on the quality of life, muscle strength, balance, and physical ability in the elderly. Rev Bras de Geriatria e Gerontologia. 2013:16(1):117-126.

12 Silva $\mathrm{A}$, et al. Equilíbrio, coordenação e agilidade de idosos submetidos à prática de exercícios físicos resistidos. Rev Bras de Medicina do Esporte. 2008:14(2):88-93.

13 Fernandes IIB, Vasconcelos KC.Análise da qualidade de vida segundo o questionário sf- 36 nos funcionários da gerência de assistêncial.[Trabalho de conclusão de curso]. Belém (PA): Centro de Ciências Biológicas e da Saúde, Graduação em Fisioterapia; 2009.

14 Leotti VB, Birck AR, Riboldi J. Comparação dos Testes de Aderência à Normalidade Kolmogorov Smirnov, Anderson-Darling, Cramer-Von Mises e ShapiroWilk por Simulação. Simpósio de Estatística Aplicada à Experimentação Agronômica, 2005.

15 Silqueira SMF. O questionário Genérico sf 36 como instrumento de mensuração da qualidade de vida relacionado a saúde. [Tese de Doutorado]. Ribeirão Preto (SP): Universdidade de São Paulo, Pós-Graduação em Enfermagem; 2005.

16 Alves RL. Qualidade de vida e excesso de peso de trabalhadores de turnos alternantes de uma empresa de mineração da região dos Inconfidentes, MG. [Dissertação de Mestrado]. Ouro preto (MG): Universidade Federal de Ouro Preto, Mestrado em nutrição; 2014.

17 Gontijo RW, Leão MRC. Effectiveness of a preventive physical therapy program for the elderly. Rev Médica de Minas Gerais. 2013:23(2):173-180.

18 Jesus DF, Silva CAF. Percepção de qualidade de vida por idosos praticantes e não praticantes de exercícios resistidos: análise do Projeto Vida Corrida. Rev Lecturas, Educación Fisica y Desportes. 2010:15(1): 34-46. 
19 Pimenta FAP, et al. Avaliação da qualidade de vida de aposentados com a utilização do questionário SF-36. Rev da Associação Médica Brasileira. 2008:54(1):55-60.

20 Farinha KO, Almeida MS, Trippo KV. Avaliação da qualidade de vida de docentes fisioterapeutas da cidade do Salvador-Bahia. Rev Pesquisa em Fisioterapia. 2003:3(1):13-35.

21 Silva ER, Gonçalves SEAL, Pompilio TG. A análise da qualidade de vida em idosos praticantes de dança de salão pelo questionário sf - 36 - araçatuba - sp. [Trabalho de conclusão de curso] Lins (SP): Centro Universitário Católico Salesiano Auxilium, Graduação em Educação Física; 2012.

22 Suzano DS, Almeida MCS, Massa LDB, Wengert M. A importância da qualidade de vida em pacientes hipertensos. Saúde em Redes. 2016:2(1): 53-63.

23 Castro $\mathrm{M}$, et al. Qualidade de vida de pacientes com insuficiência renal crônica em hemodiálise avaliada através do instrumento genérico SF-36. Rev da Associação Médica Brasileira. 2003:49(3):245-249.

24 Lopes A, Ciconelli L, Ciconelli A, Reis R, Reis F. Medidas de avaliação de qualidade de vida e estados de saúde em ortopedia. Rev Bras de ortopedia. 2007:42(11):355-359.

25 BRASIL. Lei 8.080/90.

26 BRASIL. Lei 8.142/90.

Rev. Psicol Saúde e Debate. Jan., 2017:3(1):50-67. 